\title{
DES CONSÉQUENCES MORTELLES DU CHOIX DES ACHETEURS DANS LA FILIÈRE DU BÂTIMENT. ÉTUDE DE CAS DE LA CÉRAMIQUE SANITAIRE
}

Deadly consequences of buyers' choices in the building sector. Case study of vitreous china

\section{Lise Serra \\ Université de La Réunion / PIMENT, France \\ lise.serra@univ-reunion.fr}

RÉSUMÉ : Cet article envisage la ville comme un ensemble de matériaux mis en œuvre résultant du choix de donneurs d'ordre publics et privés. Ces choix, largement inconscients, peuvent avoir des conséquences létales sur des populations de travailleurs éloignées du lieu du chantier, notamment dans le secteur d'extraction des matières premières. Ce travail vise à enrichir les options de choix des donneurs d'ordre dans le secteur du bâtiment en proposant un critère éthique basé sur le risque d'accidents mortels du travail tout au long de la filière étudiée. Les informations récoltées ont été de deux ordres. En géographie : où sont situés les principaux lieux d'extraction des minerais étudiés ? En droit international : quels sont les chiffres existants et les conventions ratifiées afin d'évaluer le risque d'accident mortel par secteur d'activité et par pays ? Les résultats présentés permettent d'envisager de façon optimiste la suite du travail. D'une part il existe plusieurs pays qui peuvent fournir les minerais de façon industrielle et les transformer dans de très bonnes conditions de sécurité et de santé des travailleurs. D'autre part, les acteurs rencontrés semblent enclins à s'engager plus loin dans la démarche pour construire une ville durable d'un point de vue tant écologique qu'éthique, respectant les droits de tous les hommes.

Mots CLÉs : ville, construction, sécurité et santé des travailleurs, céramique sanitaire. 
RESUM: Aquest article considera la ciutat com un conjunt de materials disposats en funció de l'elecció de contractistes públics i privats. Aquestes eleccions, en gran mesura inconscients, poden tenir conseqüències letals a les poblacions de treballadors allunyats del lloc de construcció, especialment al sector de l'extracció de les matèries primeres. Aquest treball té l'objectiu d'enriquir les opcions dels contractistes del sector de la construcció amb la proposta d'un criteri ètic basat al risc d'accidents laborals mortals en aquesta indústria. La informació recollida ha sigut de dos ordres. En geografia: On se situen els principals llocs d'extracció dels minerals estudiats? En dret internacional: Quines son les xifres existents i els convenis ratificats per avaluar el risc d'accidents mortals per sectors d'activitat i per país? Els resultats presentats permeten entreveure amb optimisme la continuació del treball. D'una banda existeixen diversos països que poden subministrar els minerals de manera industrial i transformar-los en molt bones condicions de seguretat i salut dels treballadors. D'altra banda, els actors consultats semblen disposats a acceptar més compromisos envers la construcció ecològica i ètica de ciutats sostenibles des del respecte als drets de tots els essers humans.

Paraules clau: ciutat, construcció, seguretat i salut dels treballadors, ceràmica sanitària.

RESUMEN: Este artículo considera a la ciudad como un conjunto de materiales dispuestos en función de la elección de contratistas públicos y privados. Estas elecciones, en gran parte inconscientes, pueden tener consecuencias letales en las poblaciones de trabajadores alejados de los solares en construcción, especialmente en el sector de la extracción de las materias primas. Este trabajo busca enriquecer las opciones de los contratistas en el sector de la construcción con la propuesta de un criterio ético basado en el riesgo de accidentes laborales mortales en esta industria. La información recopilada se orienta en dos sentidos. En geografía: ¿Dónde se sitúan los principales lugares de extracción de los minerales estudiados? En derecho internacional: ¿Cuáles son las cifras existentes y los convenios ratificados para evaluar el riesgo de accidente mortal por sector de actividad y por país? Los resultados presentados permiten encarar con optimismo el progreso del trabajo. Por un lado existen varios países que pueden proveer los minerales de forma industrial y transformarlos en muy buenas condiciones de seguridad y salud para los trabajadores. Por otra parte, los actores consultados parecen dispuestos a aceptar más compromisos en sus funciones de construcción ecológica y ética de ciudades sostenibles respetando los derechos de todos los seres humanos.

Palabras Clave: ciudad, construcción, seguridad y salud de los trabajadores, cerámica sanitaria.

ABSTRACT: This paper considers the city as a set of materials implemented as a result of choices made by public and private contractors. These largely unconscious choices can have lethal consequences on workers far from the building sites themselves, particularly in the mining sector. This research aims to expand the choice of options available to contractors in the construction sector by proposing an ethical criterion based on fatal injuries at work. Two kinds of information were gathered: geographical data on the location of the main extraction sites for the minerals studied; and international legislation on the existing figures and ratified conventions allowing assessment of the risk of fatal injuries by sector of activity and country. The results paint an optimistic picture for the future of such work. On the one hand, several countries can supply and process minerals under excellent conditions of health and safety for workers. On the other hand, the stakeholders interviewed seem open to further commitments in the process towards an ecologically and ethically sustainable city that respects everyone's rights.

KEYWORDS: city, construction, worker safety and health, sanitary ceramics. 
Tout individu a droit à la vie, à la liberté et à la sûreté de sa personne. Article 3 de la Déclaration universelle des droits de l'homme, adoptée à Paris le 10 décembre 1948 par les 58 États Membres.

\section{Introduction}

errière les façades de la ville, des milliers de mètres cubes de béton, de plâtre, de carreaux de ciment abritent les citadins. Des lits, des armoires, des baignoires, des cuvettes de toilettes sont utilisées quotidiennement par tous les foyers urbains. La ville est, dans ce sens, le lieu de concentration de matières, de matériaux et de matériels issus du monde entier. Or, beaucoup de ces éléments parcourent un circuit rythmé par des risques d'accidents du travail plus ou moins graves, voire mortels. L'information de ces risques circulant moins facilement que les matériaux eux-mêmes, les donneurs d'ordre du secteur du bâtiment font des choix en grande partie inconscients, non-informés.

La première hypothèse de cet article est que les informations sur la mondialisation des ressources planétaires (Sassen, 1999) peuvent permettre de préciser les critères de choix des donneurs d'ordre (Hämäläinena, 2009). L'hypothèse de la méthode s'appuie sur une étude de cas limitée à un matériau spécifique : la céramique sanitaire. Le croisement des données concernant la production des matières premières nécessaires à la fabrication de la céramique sanitaire, les statistiques de l'Organisation Internationale du Travail (OIT) et la ratification du Protocole facultatif se rapportant au Pacte international relatif aux droits économiques, sociaux et culturels adopté par l'Assemblée générale des Nations unies en 2008 doit permettre d'évaluer le risque d'accident mortel lié à l'extraction du minerai au sein des pays producteurs.

Le premier objectif de cette étude est de sensibiliser les acteurs du bâtiment quant à l'importance en termes d'éthique individuelle et collective des choix émis à chaque étape de la conception et de la construction. Le deuxième objectif est de mettre en place les pistes de recherche pour envisager de faire évoluer durablement le secteur du bâtiment afin de prendre en compte toutes les vies humaines à un même niveau d'égalité. En effet, si la protection de l'environnement est aujourd'hui bien prise en compte dans les modes constructifs contemporains, la valeur de toutes les vies humaines est encore loin des préoccupations des acteurs de la filière.

\section{Méthode}

Au sein du domaine de recherche lié à l'analyse du cycle de vie puis à l'analyse sociale du cycle de vie (Guinée, 2011), les travaux se partagent entre des analyses par filières, par matériaux spécifiques et des analyses méthodologiques pour améliorer la fiabilité de ces analyses (Grubert, 2018). Je souhaite proposer ici de limiter l'analyse à la fois par le type de matériau étudié et le type de critère analysé. L'analyse du cycle de vie, telle que fondée dans les années 1960 (Smith, 1963), s'appuie principalement sur un bilan carbone statistique avec l'objectif de comparer des matériaux et des objets entre eux. Dès 1975, Paul A. Samuelson propose d'évaluer la sécurité sociale au sein du modèle d'analyse du cycle de vie (Samuelson, 1975). Ces premières propositions ont ensuite donné lieu à des travaux réguliers et un journal spécialisé, The international journal of life cycle assessment. Cependant, ces travaux restent encore trop réservés au domaine scientifique ou à quelques entreprises qui développent de la recherche et développement en interne. La majorité des donneurs d'ordre du bâtiment n'a pas accès à ces questionnements, par manque de vulgarisation de la part du milieu scientifique et par manque de temps de leur part.

L'hypothèse soumise dans cet article repose sur la valeur de la vie d'un homme comme élément trop important pour être relativisé et compris comme un point statistique parmi d'autres, permettant de construire une moyenne visant à la comparaison entre matériaux. Le risque d'accident mortel au travail est donc le seul critère étudié ici. La méthode d'évaluation de la sécurité et de la santé au travail fait l'objet d'une des 33 fiches du guide Methodological sheets for sub-categories in social life cycle assessment (Benoît Norris, Traverso, 2013). L'évaluation et la réduction des risques professionnels par la surveillance et l'amélioration du milieu de travail font partie du Plan d'action 
mondial pour la santé des travailleurs porté par l'Assemblée mondiale de la santé (résolution WHA60.26, 2007).

Ensuite, parmi la grande diversité des matériaux mis en œuvre dans un bâtiment, le béton, l'acier, la brique ou le bois sont les éléments les plus importants en termes de volume de matière. Cependant, ces éléments sont aussi les mieux connus, d'une part, et pour certains, représentant les filières les plus opaques. Par exemple, en 2013, Le Monde, journal français, décrit le trafic illégal et criminel de sable à grande échelle qui sévit en Inde. L'éditeur lance la même année le projet " écocide " " pour désigner les trafics illicites perpétrés par le crime organisé » (Bouissou, 2015). En 2017, c'est le Maroc qui est à l'honneur du journal où « des filières légales et clandestines se disputent le sable nécessaire à la confection du béton. Le trafic est tel que des plages entières sont menacées de disparition. » (Kadiri, 2017). En 2018, ONU Environnement, GRID (base de données sur les ressources mondiales), la ville de Genève et l'Université de Genève organisent la première table ronde sur le sable. Le matériau sable est donc déjà traité partiellement et le travail encore nécessaire est colossal.

D'autres éléments composent un habitat standard : matériaux de second œuvre, mobilier, mobilier sanitaire. Le choix de l'étude de cas concerne la céramique sanitaire, produit spécifique de la filière céramique. Cela permet en premier lieu une approche symbolique de l'analyse du cycle de vie. Est-on d'accord de se laver tous les matins dans un lavabo qui suppose, peut-être, la mort d'un homme ? Au-delà de cette question symbolique, la céramique sanitaire est composée de trois minerais principaux : le kaolin, à hauteur de $50 \%$, le feldspath et le quartz, chacun à hauteur de $25 \%$. Le nombre réduit d'ingrédients permet une recherche limitée dans le temps afin d'aboutir à la première carte ci-dessous des principaux pays producteurs de ces minerais. Les cartes sont réalisées par Alexandre Fays, étudiant stagiaire au sein du laboratoire PIMENT entre mai et juin 2019.

La question posée en introduction suppose également de connaître le risque d'accidents mortels durant l'extraction de ces minerais. L'ort propose un classement du nombre de morts par année, par pays et par secteur économique. Ces chiffres sont issus des données des administrations d'États et des sociétés d'assurances et couvrent l'ensemble des travailleurs assurés.
Les travailleurs en situation illégale sont donc absents de ces statistiques. La liste des pays dont l'ort possède les données est limitée et ne recouvre pas tous les principaux pays producteurs de kaolin, de feldspath et de quartz. Une deuxième recherche a donc porté sur les conventions, pactes et protocoles internationaux existants concernant la protection de la sécurité et de la santé des travailleurs. En décembre 1966, le Pacte international relatif aux droits économiques, sociaux et culturels est adopté par l'Assemblée générale des Nations unies et entre en vigueur en janvier 1976. L'article 12 prend en compte le droit de jouir d'un meilleur état de santé, de sécurité sanitaire et d'une couverture maladie universelle. En 2016, ce pacte était ratifié par 164 États dont la très grande majorité des pays producteurs des minerais étudiés ici. En 1981, l' Oाт adopte la convention sur la sécurité et la santé au travail. En 2015 cette convention est ratifiée par 33 pays. En 2002, un protocole relatif à la convention sur la sécurité et la santé au travail est adopté par l' OIT. Ce protocole est ratifié par 12 pays. Le 18 juin 2008, l'Assemblée générale des Nations unies adopte le Protocole facultatif se rapportant au Pacte international relatif aux droits économiques, sociaux et culturels. Le Comité des droits économiques, sociaux et culturels est alors habilité à examiner les cas de violation du Pacte international relatif aux droits économiques, sociaux et culturels. La ratification de ce protocole et sa signature par 45 États permet une application concrète et contextualisée du pacte adopté 42 ans plus tôt. Dans ce travail de recherche, seront retenus uniquement les traités des Nations unies, rassemblant plus largement la communauté internationale. Les analyses par pays du risque d'accident mortel sont reprises dans la deuxième carte présentée ci-dessous.

Enfin, pour compléter le protocole méthodologique de ce travail de recherche préliminaire, trois entretiens ont été menés à La Réunion, Département français d'Outre-mer où je suis située : le lundi 27 mai 2019 auprès d'un responsable de projet d'un bailleur public, le mercredi 29 mai auprès d'un responsable des marchés publics d'une municipalité et le lundi 3 juin auprès d'un gérant d'une société de plomberie. Ces entretiens ont permis de présenter le projet de recherche et d'évaluer la réception des hypothèses par ces acteurs du marché du bâtiment. La vie d'un homme leur parait-elle être un critère suffisant pour faire évoluer les pratiques de la commande publique et privée actuelle? À quel prix les collectivités et entreprises qu'ils représentent 
seraient prêtes à s'engager pour défendre la vie d'un homme, à l'autre bout du monde, dont la mort reste hypothétiquement liée à leur choix final en tant qu'acheteur? Quelles seraient les conditions nécessaires pour faire évoluer, à leur niveau, leurs habitudes?

\section{Résultats}

\section{Processus de fabrication}

Les objets sanitaires en céramique vitrifiée sont produits par moulage et double cuisson d'une pâte céramique issue principalement des minerais de kaolin, de quartz (silice cristalline) et de feldspath. Le kaolin est un matériau argileux silicaté largement répandu, extrait en mines à ciel ouvert. Un gisement de kaolin peut se trouver sous la forme de poches de plus ou moins grande extension au sein des granites (Cornouailles, Bretagne, Bavière, etc.) ou avoir été déplacé. Les géologues parlent alors d'argile kaolinique que l'on trouve dans un environnement sédimentaire (Drôme, Brésil...). L'argile (kaolin) meuble est extraite par pelle mécanique et transportée par camions miniers à l'usine de préparation. L'argile (kaolin) est mise en solution, débarrassée de ses éléments grossiers par criblage, décantée, filtrée et séchée. Le kaolin entre dans la fabrication de la céramique mais également dans les industries du caoutchouc et du papier. Le quartz est un minerai très abondant, principalement extrait en mines à ciel ouvert. On trouve la silice de haute qualité soit sous forme meuble soit sous forme de «veines» de quartz dont l'épaisseur peut atteindre plusieurs mètres. Les gisements de sable de silice sont normalement exploités en carrière. Des engins de type bulldozers retournent la terre pour exposer le quartz. Les explosifs sont peu utilisés. Une fois le quartz exposé, les équipes à pied l'extraient à l'aide de pioches et de burins. Le quartz est ensuite lavé, trié puis concassé selon les usages prévus. Le matériau extrait subit parfois un important traitement avant sa commercialisation. Certains types de quartz, considérés comme semi-précieux, sont utilisés en joaillerie. La majorité du volume extrait entre dans la fabrication du verre et de la céramique. Les feldspaths sont présents dans les roches magmatiques et sont considérés comme abondants. L'extraction se fait dans des mines à ciel ouvert. L'abatage est le plus souvent réalisé à l'explosif. Le minerai extrait est stocké, concassé, séché et broyé pour l'obtention de poudres. Les industries céramiques et verrières constituent les principaux débouchés du feldspath (Pouliquen, 2009).

Les minerais broyés sont mélangés au sein des usines de production de céramique sanitaire pour l'obtention de la pâte céramique, ou barbotine, qui est ensuite moulée, cuite une première fois puis recuite et vitrifiée. La barbotine est un mélange des trois produits, largement sur la base de l'expérience du fabricant. La qualité finale des produits dépend de cette recette qui peut prendre en compte des minerais de différents gisements pour obtenir le fini souhaité. La valeur économique de ces recettes tend à les conserver secrètes, créant un premier frein à la traçabilité du point de vue de l'observateur extérieur.

Le schéma ci-dessous présente le processus d'extraction, de fabrication, de distribution et de mise en œuvre des éléments de céramique sanitaire (Commission européenne, 2007, p. 11).

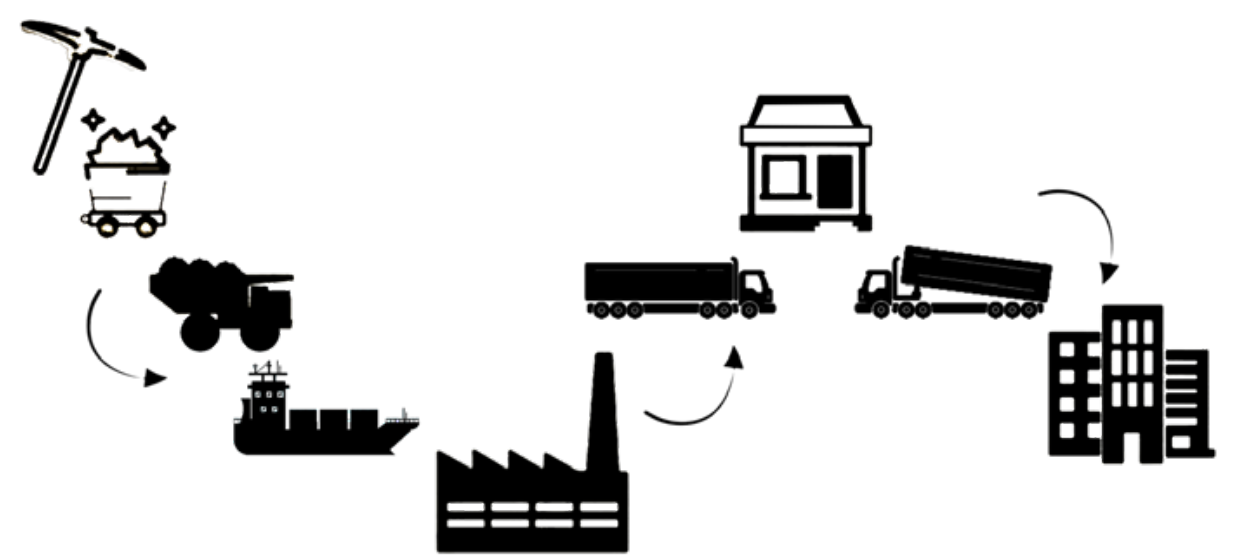

Figure 1 : Schéma du processus d'extraction, fabrication, distribution et mise en ouvre des éléments de céramique sanitaire. Source : auteure

En termes d'analyse de risque, le transport est exclu de l'analyse par filière, les risques étant transversaux à l'ensemble du marché économique planétaire. Dans l'Union européenne, la mise en œuvre sur chantiers présente des risques limités et dont la dangerosité à fortement diminué au cours des décennies passées. La distribution des matériaux présente un risque très faible d'accident mortel lié à l'activité d'achat/vente. En usine, les matériaux 
dégagent une fine poussière susceptible d'être nocive. Le port du masque suffit à assurer la santé des travailleurs. La cuisson à haute température constitue une ambiance qui s'apparente à celle des fonderies. Les problèmes inhérents à toutes les industries de haute température constituent les principaux risques d'accidents au moment de la fabrication (Stellman, 2002, p. 84.17). Les évolutions du droit du travail et de son application dans les pays d'Europe ont permis de réduire considérablement les accidents mortels. Les usines présentes hors de l'Union Européenne doivent être analysées individuellement. La phase d'extraction reste donc la phase la plus dangereuse du processus en termes de risques d'accident du travail. La circulation d'engins de très grandes dimensions et l'usage d'explosifs représentent les principaux risques d'accidents. Cette phase d'extraction est d'ailleurs non référencée dans le Document de référence sur les meilleures techniques de fabrication de céramique proposé par la Commission européenne (2007).

\begin{tabular}{|l|r|r|l|r|l|l|l|l|l|l|}
\hline \multicolumn{7}{|c|}{ Classement des pays producteurs } \\
\hline \multicolumn{7}{|c|}{ Production de kaolin supérieure ou égale à 100 000 tonnes/an (2007) } \\
\hline 1 & États-Unis & 7 & Ukraine & 13 & Turquie & 19 & Égypte & 25 & Jordanie \\
\hline 2 & Ouzbékistan & 8 & Royaume Uni & 14 & Espagne & 20 & Belgique & 26 & Algérie \\
\hline 3 & Rép. Tchèque & 9 & Bulgarie & 15 & Kirghizistan & 21 & Australie & 27 & Nigeria \\
\hline 4 & Allemagne & 10 & Mexique & 16 & Iran & 22 & Pologne & & \\
\hline 5 & Corée du Sud & 11 & Vietnam & 17 & Malaisie & 23 & Thaïlande & & \\
\hline 6 & Brésil & 12 & Italie & 18 & France & 24 & Portugal & & \\
\hline Production de feldspath supérieure ou égale à 50 000 tonnes/an (2008) & & \\
\hline 1 & Turquie & 7 & État Unis & 13 & Inde & 19 & Vénézuela & 25 & Bulgarie \\
\hline 2 & Italie & 8 & France & 14 & Portugal & 20 & Brésil & 26 & Norvège \\
\hline 3 & Chine & 9 & Rép. Tchèque & 15 & Égypte & 21 & Allemagne & 27 & Finlande \\
\hline 4 & Japon & 10 & Pologne & 16 & Malaisie & 22 & Afrique du Sud & 28 & Australie \\
\hline 5 & Thaïlande & 11 & Mexique & 17 & Iran & 23 & Grèce & & \\
\hline 6 & Espagne & 12 & Corée de Sud & 18 & Argentine & 24 & Colombie & & \\
\hline $\mathbf{1 5}$ & principaux pays producteurs de quartz (2017) & & & \\
\hline 1 & Chine & 4 & Espagne & 7 & Allemagne & 10 & Égypte & 13 & Sri Lanka \\
\hline 2 & Turquie & 5 & Brésil & 8 & Italie & 11 & Norvège & 14 & Belgique \\
\hline 3 & Inde & 6 & États-Unis & 9 & Canada & 12 & Ukraine & 15 & Russie \\
\hline
\end{tabular}

Figure 2 : Classement des pays selon leur production. Sources : https://www. indexmundi.com $;$ https://www.worldatlas.com

\section{Répartition géographique}

Les principaux pays producteurs de kaolin, de feldspath et de quartz, sont classés dans le tableau ci-dessous par importance en volume d'exportation.

Les pays producteurs sont référencés dans la carte mondiale ci-dessous, permettant de constater la répartition des mines sur tous les continents.

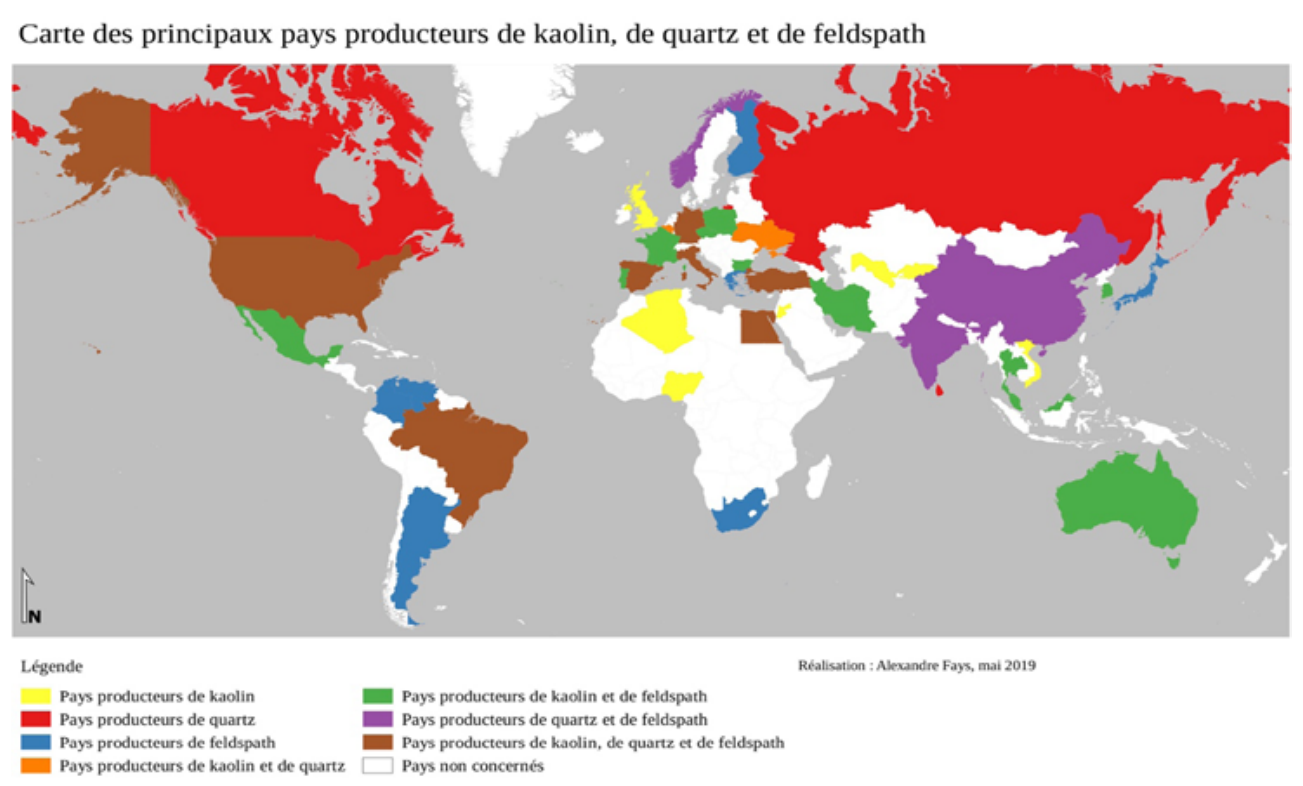

Figure 3 : Carte des principaux pays producteurs de kaolin, feldspath et quartz. Sources des données cartographiées : http://www.zafermaden.com; https://www.mineraliindustriali.it; https://www.geologyforinvestors.com; https://www.usgs.gov; http://www.strategicminerals.com; http://mineral.com ua/en/; https://pubs.geoscienceworld.org; https://search.gmdu.net; http://www. turkishminerals.org; https://imerys-kaolin.com; https://www.minfind.com; turkishminerals.org; https://imerys-kaolin.com; https://www.minfind.com;
http://www.kaltun.com.tr; http://www.mahavirminerals.com; https://www. mindat.orgl; https://www.ilo.org; http://indicators.ohchr.org

Les pays représentés en jaune sont uniquement producteurs de kaolin, en rouge de quartz et en bleu de feldspath. Les couleurs orange, vert et violet correspondent aux pays producteurs de deux types de minerai et la couleur marron correspond aux pays qui produisent, de façon industrielle, les trois minerais nécessaires à la fabrication de céramique sanitaire.

La carte suivante présente, dans les tons orangés, les chiffres de l'ort concernant le nombre de morts annuel moyen pour 100000 travailleurs dans le secteur minier entre 2008 et 2017. Pour les pays non référencés par l' оाт, la 
signature du pacte relatif aux droits économiques, sociaux et culturels adopté en 1966 puis la ratification du protocole optionnel relatif à ce pacte, adopté par l'Assemblée générale des Nations unies en 2008, présente des éléments d'analyse pour évaluer le risque d'accident mortel au sein de chaque pays producteur des minerais entrant dans la composition de la céramique sanitaire.

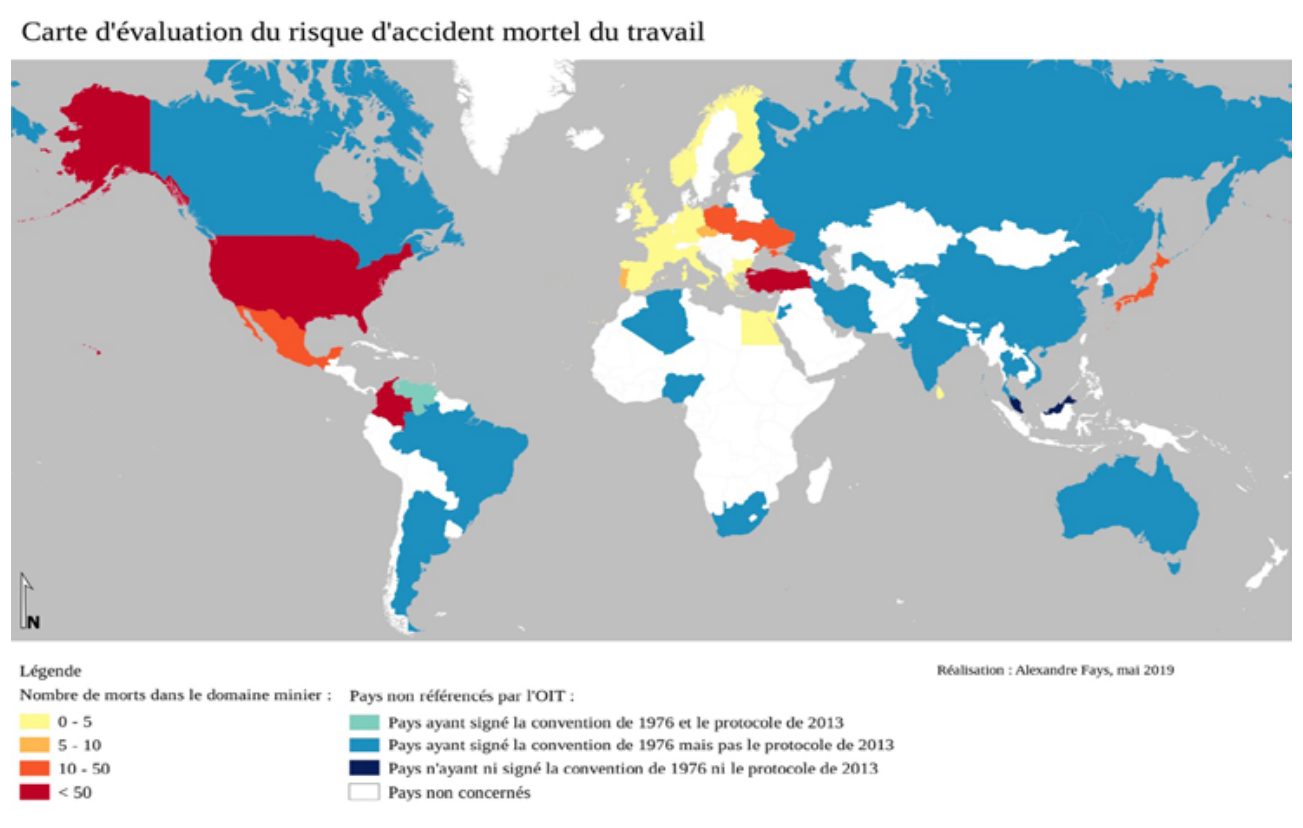

Figure 4 : Carte d'évaluation du risque d'accident mortel au travail dans les pays producteurs de kaolin, feldspath et quartz. Sources des données cartographiées https://www.ilo.org; http://indicators.ohchr.org/

Les bais de cette carte concernent principalement l'étendue du secteur minier, comprenant l'ensemble des mines d'extraction de minerais précieux et semi précieux autant que des minerais plus courants. Les mines sous-terraines et les mines à ciel ouvert sont englobées dans le même ensemble statistique. Ainsi, avant d'avoir des informations plus précises concernant spécifiquement les mines de kaolin, de feldspath et de quartz, cette carte permet uniquement d'apprécier le risque d'accident mortel dans le secteur minier à l'échelle d'un pays. Cela signifie que les pays qui présentent, dans leur globalité, un nombre de morts très faibles, peuvent être considérés comme respectueux du droit à la vie de tous les hommes travaillant légalement sur leur sol. Cela ne signifie pas que dans les pays présentant des taux de mortalité élevé, les travailleurs embauchés dans les mines de kaolin, de feldspath ou de quartz soient plus exposés à un risque d'accident mortel. Cela signifie uniquement que, au niveau des États, les engagements ne sont pas encore pris pour orienter toutes les pratiques vers le risque létal zéro.

Le tableau suivant reprend synthétiquement, pour l'ensemble des pays producteurs des minerais concernés :

- La population du pays ;

- Le volume en tonnes par type de minerai produit et le total en milliers de tonnes pour les trois types de minerais ;

- Le nombre moyen annuel de morts dans le secteur minier, par pays, entre 2008 et 2017 selon l'orT ;

- Le nombre moyen annuel de morts pour 100000 travailleurs dans le secteur minier, par pays, entre 2008 et 2017 selon l' OIT ;

- La date de signature par les États du Pacte international des droits économiques, sociaux et culturel (1976) ;

- La date de signature par les États du Protocole optionnel associé (2013)

NS signifie « non signé ».

\begin{tabular}{|c|c|c|c|c|c|c|c|c|c|}
\hline \multirow{2}{*}{ PAYS } & \multirow{2}{*}{$\begin{array}{l}\text { Population } \\
\text { en millions } \\
\text { d'hab. }\end{array}$} & \multicolumn{3}{|c|}{$\begin{array}{l}\text { Volume d'extraction par } \\
\text { minéraux, en tonnes }\end{array}$} & \multirow{2}{*}{$\begin{array}{c}\text { Volume } \\
\text { total d'ex- } \\
\text { traction, en } \\
\text { milliers de } \\
\text { tonnes }\end{array}$} & \multirow{2}{*}{$\begin{array}{c}\text { Nombre } \\
\text { de morts } \\
\text { annuel } \\
\text { absolu }\end{array}$} & \multirow{2}{*}{$\begin{array}{c}\text { Nombre } \\
\text { de morts } \\
\text { annuel } \\
\text { pour } \\
100000 \\
\text { travai- } \\
\text { Ileurs }\end{array}$} & \multirow{2}{*}{$\begin{array}{c}\text { Ratification } \\
\text { de la con- } \\
\text { vention de } \\
1976\end{array}$} & \multirow{2}{*}{$\begin{array}{l}\text { Ratifi- } \\
\text { cation du } \\
\text { protocole } \\
\text { de } 2013\end{array}$} \\
\hline & & Feldspath & Kaolin & Quartz & & & & & \\
\hline Afrique du Sud & 54,96 & 105815 & & & 106 & 3,5 & ' & 2015 & NS \\
\hline Algérie & 41,10 & & 106567 & & 107 & 1 & 1 & 1989 & NS \\
\hline Allemagne & 82,80 & 170000 & 3800000 & 305291 & 4275 & 5,3 & 4,9 & 1973 & NS \\
\hline Argentine & 43,59 & 291562 & & & 292 & 1 & 1 & 1986 & 2011 \\
\hline Australie & 24,00 & 50000 & 250000 & & 300 & 1 & 1 & 1975 & NS \\
\hline Belgique & 11,29 & & 300000 & 127873 & 428 & 0,3 & 10,9 & 1983 & 2014 \\
\hline Brésil & 206,98 & 172000 & 2500000 & 483836 & 3156 & 47 & 21,1 & 1992 & NS \\
\hline Bulgarie & 7,15 & 90000 & 1631000 & & 1721 & 5 & 15,6 & 1970 & NS \\
\hline Canada & 36,16 & & & 222273 & 222 & 1 & 1 & 1976 & NS \\
\hline Chine & 1364,64 & 2000000 & & 1314345 & 3314 & 1 & 1 & 2001 & NS \\
\hline Colombie & 48,53 & 92000 & & & 92 & 53,5 & 26,4 & 1969 & NS \\
\hline Corée du Sud & 51,54 & 400000 & 2630356 & & 3030 & / & / & 1990 & NS \\
\hline
\end{tabular}




\begin{tabular}{|c|c|c|c|c|c|c|c|c|c|}
\hline Égypte & 97,44 & 360000 & 300000 & 185527 & 846 & 2 & 36,1 & 1982 & NS \\
\hline Espagne & 46,44 & 675000 & 450000 & 492855 & 1618 & 6,6 & 25,9 & 1977 & 2010 \\
\hline États-Unis & 334,52 & 650000 & 7110000 & 388200 & 8148 & 147,9 & 15,4 & $\begin{array}{l}\text { signé en } \\
1977 \text { mais } \\
\text { non ratifíe }\end{array}$ & NS \\
\hline Finlande & 5,50 & 60000 & & & 60 & 0,5 & 10,9 & 1975 & 2014 \\
\hline France & 69,86 & 650000 & 307253 & & 957 & 2,3 & 6,5 & 1980 & 2015 \\
\hline Grèce & 10,79 & 95000 & & & 95 & 1,7 & 15,7 & 1985 & NS \\
\hline Inde & 1364,63 & 400000 & & 796855 & 1197 & I & 1 & 1979 & NS \\
\hline Iran & 82,80 & 300000 & 350000 & & 650 & I & 1 & 1975 & NS \\
\hline Italie & 61,30 & 4727000 & 584121 & 240345 & 5551 & 5,5 & 16,4 & 1978 & 2015 \\
\hline Japon & 124,86 & 700000 & & & 700 & 9,7 & 39 & 1979 & NS \\
\hline Jordanie & 6,71 & & 110000 & & 110 & I & 1 & 1975 & NS \\
\hline Kirghizistan & 5,90 & & 400000 & & 400 & 5,7 & 50,8 & 1994 & NS \\
\hline Malaisie & 30,42 & 300000 & 350000 & & 650 & 1 & 1 & NS & NS \\
\hline Mexique & 123,58 & 432840 & 962000 & & 1395 & 32 & 24,2 & 1981 & NS \\
\hline Nigeria & 198,94 & & 100000 & & 100 & I & I & 1993 & NS \\
\hline Norvège & 5,21 & 75000 & & 185109 & 260 & 1,6 & 2,4 & 1972 & NS \\
\hline Ouzbékistan & 30,49 & & 5500000 & & 5500 & I & 1 & 1995 & NS \\
\hline Pologne & 37,97 & 440000 & 210373 & & 650 & 26,6 & 13,5 & 1977 & NS \\
\hline Portugal & 10,34 & 372000 & 160000 & & 532 & 5,1 & 34,6 & 1978 & 2013 \\
\hline Rép. Tchèque & 10,45 & 510000 & 3800000 & & 4310 & 6 & 14,7 & 1993 & NS \\
\hline Royaume Uni & 63,42 & & 1800000 & & 1800 & 4,1 & 6,2 & 1976 & NS \\
\hline Russie & 126,43 & & & 125927 & 126 & 128 & 13 & 1973 & NS \\
\hline Sri Lanka & 20,68 & & & 156764 & 157 & 3,25 & 5,5 & 1980 & NS \\
\hline Thailande & 61,38 & 678000 & 200000 & & 878 & 8,5 & 1 & 1999 & NS \\
\hline Turquie & 82,80 & 6500000 & 580000 & 1239291 & 8319 & 111,4 & 75,8 & 2003 & NS \\
\hline Ukraine & 42,77 & & 2386000 & 163982 & 2550 & 49,4 & 15,4 & 1973 & $\begin{array}{l}\text { ignné ne } \\
2009 \\
\text { non ratifi }\end{array}$ \\
\hline Vénézuela & 30,21 & 200000 & & & 200 & I & 1 & 1978 & 2018 \\
\hline Vietnam & 91,70 & & 650000 & & 650 & I & 1 & 1982 & NS \\
\hline
\end{tabular}

Figure 5 : Synthèse par pays du type de production, du nombre de morts dans le secteur minier selon l'ort et des dates de signature du Pacte de 1976 et du Protocole de 2013 sur les droits économiques, sociaux et culturels. Sources des données : Wikipédia pour les données de populations, indexmundi.com pour les données concernant le feldspath et le kaolin, worldatlas.com pour les données concernant le quartz, ilo.org pour les données de l'ort.
Le deuxième biais qui apparaît à la lecture de ce tableau concerne la qualité des chiffres de l'ort. Ces chiffres sont issus de déclarations administratives des États et des sociétés d'assurance. Les groupes de référence concernent principalement les personnes assurées, en situation de travail légal. Le trafic illégal et l'emploi non déclaré n'apparaissent donc pas ici. De plus, les chiffres prennent en compte, de façon variable, la survenue d'accidents mortels sur le trajet domicile-travail. Enfin, il aurait été intéressant de connaître le nombre total de morts dans le secteur minier pour l'ensemble des pays mais les données disponibles sont trop incomplètes pour construire une véritable vision d'ensemble.

\section{Discussion}

Des pays producteurs dans le monde entier mais inégaux face aux risques d'accidents mortels

En volume total d'extraction, la Turquie et les États-Unis arrivent en tête avec chacun plus de huit millions de tonnes de feldspath, kaolin et quartz extraits annuellement. L'Italie, l'Ouzbékistan, la République Tchèque et l'Allemagne suivent avec plus de quatre millions de tonnes chacun. Ces pays grands producteurs sont répartis sur trois continents représentant une couverture mondiale globale.

Les plus gros producteurs présentent des caractéristiques inégales en termes de nombre de morts annuels dans le secteur minier. La Turquie est le pays le plus touché par rapport aux données dont nous disposons, présentant un triste total de 111,4 morts en moyenne annuelle, soit 75,8 morts pour 100000 travailleurs par an dans le secteur minier. Les États-Unis présentent également un total assez négatif avec 147,9 morts en chiffre brut, équivalent à 15,4 morts pour 100000 travailleurs. L'Italie est $15^{\mathrm{e}}$ sur notre classement en nombre de morts pour 100000 travailleurs, la République Tchèque $10^{\mathrm{e}}$, 1'Allemagne $2^{e}$ et l'Ouzbékistan n'est pas référencé par l'OІт. Les risques de mortalité sont très divers, montrant l'absence de lien entre dangerosité du secteur économique minier et gestion du risque fatal. Le cas de l'Allemagne apporte la preuve qu'il est possible de produire du minerai de façon industrielle sans mettre en danger les travailleurs de ce secteur. 


\section{Des pays bons élèves dans le secteur minier}

Les cartes et le tableau de synthèse mettent en avant un certain nombre de pays producteurs de minerai nécessaires à la production de céramique sanitaire et présentant un taux de mortalité dans le secteur minier très bas. Ainsi, la Norvège, l'Allemagne, le Sri Lanka, le Royaume Uni et la France présentent, entre 2008 et 2017, un nombre annuel moyen de morts pour 100000 travailleurs inférieur à 10 , tout en proposant une extraction industrielle des matériaux concernés. On peut ajouter à cette liste des « bons élèves » la Belgique, la Finlande, la Grèce et l'Égypte qui présentent moins de trois cas d'accidents mortels en moyenne par an. Le faible nombre d'employés dans ce secteur pour ces quatre pays explique un taux de mortalité rapporté à 100000 travailleurs assez élevé.

Parmi ces dix pays qui arrivent en tête du classement sur les 26 pays pour lesquels nous disposons de données, seules la Belgique, la Finlande et la France sont signataires du protocole optionnel lié au Pacte relatif aux droits économiques sociaux et culturels des Nations unies.

Ces résultats permettent d'envisager positivement l'avenir. En effet, l'extraction minière n'est plus un secteur structurellement mortel puisque, lorsqu'on revient aux tables détaillées par années de l'ort, un certain nombre de pays présentent un bilan nul à la fin de l'année et plusieurs années de suite. Sur les dix ans couverts par les données de l'ort, au minimum un ou deux accidents mortels sont survenus, interdisant pour l'instant d'envisager le risque zéro. Ce risque nuit à l'image du secteur.

\section{Des pays à mortalité élevée dans le secteur minier}

Au contraire, le Kirghizistan $(50,8)$ et la Turquie $(75,8)$ présentent un taux très élevé de mortalité dans le secteur minier, au-delà de 50 morts pour 100000 travailleurs. La Colombie (53,5), la Russie (128) et les États-Unis $(147,9)$ présentent également un nombre de morts absolus par an très élevé, bien que, rapporté à 100000 travailleurs le taux soit plus faible. Aucun de ces pays n'est signataire du protocole optionnel lié au Pacte relatif aux droits économiques sociaux et culturels des Nations unies. Malgré le peu de précision des données, ces chiffres montrent un risque important de mortalité dans ces pays, permettant aux donneurs d'ordre d'éviter pour l'instant les matériaux issus de ces pays, dans l'attente d'une amélioration importante et globale des conditions d'extraction.

\section{La filière de la céramique industrielle représentative d'un secteur minier en} évolution

Les États-Unis, l'Espagne et l'Italie sont des pays reconnus dans la production industrielle de matériaux de construction, notamment en termes de céramique. Le groupe familial espagnol Roca est, en 1999, à la deuxième place sur le marché mondial derrière American standard (Usine nouvelle, 1999). Ces trois pays sont des producteurs importants des trois types de minerais mais présentent un taux de mortalité annuel élevé $(15,4$ pour les États-Unis, 16,4 pour l'Italie, 25,9 pour l'Espagne). La filière céramique peut donc être considérée comme une filière à risque et les donneurs d'ordre ont actuellement peu de choix pour envisager des constructions respectant réellement les droits de tous. En effet, ne pas faire appel aux majors du secteur économique représente un travail de recherche plus important. Les donneurs d'ordre doivent se tourner vers des entreprises plus petites, moins reconnues, présentant des catalogues moins fournis et un débit moins important mais pouvant permettre un meilleur dialogue et, peut-être, une plus grande traçabilité de l'ensemble de leur filière. De plus, les certifications de fabrication européenne, par exemple, ne sont pas non plus satisfaisantes puisque de nombreux pays européens présentent encore des taux de mortalité élevés. La provenance depuis ces pays devient un critère non satisfaisant de choix pour prétendre à une filière de construction qui prenne en compte les droits universels de tous les hommes à la vie et à la santé au travail. Les producteurs de mobilier sanitaire devraient alors décrire, de façon plus détaillée, l'origine des matériaux et produire les données chiffrées concernant les taux de mortalité à toutes les étapes de production pour répondre à un donneur d'ordre exigeant en termes d'éthique constructive et de respect du droit de tous les hommes.

\section{Un pacte et un protocole peu représentatifs aujourd'hui}

Si la convention de 1976 est largement ratifiée par l'ensemble des États, le Protocole optionnel de 2013 l'est beaucoup moins. De plus, les pays signataires et non signataires de ce protocole présentent des taux de mortalité très disparates. Ainsi, les quatre premiers pays en termes de faible taux 
de mortalité (Norvège, Allemagne, Sri Lanka et Royaume Uni) ne sont pas signataires du Protocole. À l'inverse, le Portugal et l'Espagne, ont signé ce Protocole mais présentent des taux de mortalité supérieurs à 25 morts pour 100000 travailleurs par an dans le secteur minier entre 2008 et 2017. En l'absence de données de l'orт, le critère de signature du protocole optionnel ne permet donc pas d'évaluer de façon pertinente le risque d'accident mortel lié aux conditions de travail dans un pays en particulier, même si on peut espérer que les évolutions en cours sont positives. Le Protocole optionnel est encore récent et pourra démontrer de son utilité dans les années à venir.

\section{Pistes}

\section{Responsabilité sociale et environnementale}

Les principales sociétés européennes d'extraction de minerai sont cotées en bourse. Pour elles, les accidents mortels sont considérés comme gravissimes et restent relativement rares (entre 0 et 5 accidents par an). Elles ont l'obligation de publier des rapports RSE, de Responsabilité sociale et environnementale, affichant les mesures de sécurité en cours et à venir pour réduire encore le risque d'accidents mortels et non-mortels.

La responsabilité sociale se double d'une responsabilité environnementale. Le secteur minier a un impact sévère sur l'environnement qui est régulièrement dénoncé, notamment lors de l'ouverture de nouvelles carrières ou mines. Cette problématique risquant parfois de réduire la croissance économique d'une entreprise face à un marché concurrentiel, on peut envisager que des évolutions positives en termes de processus d'extraction soient en cours, visant à mieux respecter l'environnement et, de ce fait, protégeant plus certainement les travailleurs du secteur. C'est une première piste d'amélioration du risque d'accident dans le secteur minier.

\section{Des labels pour protéger la vie des travailleurs}

Les labels les plus utilisés actuellement dans le secteur du bâtiment protègent principalement l'environnement planétaire à travers la diminution des émissions de $\mathrm{CO}_{2}$ (labels Nature plus, Ange Bleu, Écolabel européen). L'envi- ronnement direct des constructions est également protégé à travers la certification Green building sustainable ou la démarche HQE. Les acteurs protégés en termes de santé sont principalement les usagers du bâtiment (démarche HQE, BEAM, certification LEED, label Green mark, etc.) et les travailleurs du chantier de construction, sur le site de construction (démarche indienne GRIHA, référentiels LEED et NAHB) (Coeudevez, Déoux, 2011). Actuellement, aucun label ne s'applique à protéger la qualité de vie des travailleurs sur l'ensemble de la filière du bâtiment. La qualité de vie des travailleurs fait également partie des préoccupations de l'Organisation des Nations Unies, intégrée au Pacte Mondial lancé en 2000 visant à inciter les entreprises à adopter une attitude socialement responsable à travers 10 principes fondateurs et aux 17 Objectifs de Développement Durable lancés en septembre 2015 à destination de tous, collectivités, entreprises et individus. Elle représente un des critères de l'analyse sociale du cycle de vie telle que fondée par l'ONU (O’Brien, 1996 ; Benoît Norris, 2013). Mais ces analyses constituent un travail de longue haleine appliqué jusqu'à maintenant à très peu de matériaux.

La possibilité d'un label soulignant uniquement la quasi absence de risque mortel au cours du processus d'extraction, de transformation, de fabrication et de mise en œuvre des matériaux et matériels pourrait permettre aux maitres d'ouvrage de s'engager à respecter l'article 3 de la déclaration universelle des droits de l'homme.

\section{Constitution de bases de données accessibles à tous}

Les donneurs d'ordre publics, en charge notamment de la construction des logements sociaux, des établissements scolaires et hospitaliers, doivent répondre, en France, au code des marchés publics et, en Europe, au principe de libre concurrence. La discrimination d'un matériau par rapport à sa situation géographique représente une violation de la libre concurrence. Dans ce cas, les données par pays d'extraction peuvent difficilement être intégrées dans les cahiers des charges de marchés publics. Le risque d'accident mortel au cours du processus d'extraction, de transformation, de fabrication et de mise en œuvre peut être envisagé comme un critère pertinent. Les entreprises devraient alors justifier du respect de ce critère en ayant recours à une base 
de donnée libre d'accès, régulièrement mise à jour, présentant les différentes caractéristiques de chaque matériaux en termes de risque mortel lié. Ces bases de données n'existent pas actuellement.

\section{Nécessité d'une étude de marché poussée}

Le prix constitue un des critères principaux dans le choix des matériaux et matériels du bâtiment. Cependant, lorsque la différence de prix est faible entre ce dont le maitre d'ouvrage a déjà l'habitude et la proposition nouvelle, la valeur financière peut devenir secondaire par rapport à la valeur humaniste revendiquée à travers le refus complet de la mort d'un homme dans le processus de construction. Sur certains projets plus médiatisés ou plus symboliques, comme la construction d'une école par exemple, un élu local pourrait s'engager sur le respect des Droits universels de l'homme et communiquer sur son action s'il connaît le prix à payer et si ce prix n'est pas trop élevé.

\section{Expérimentation en marché public et en marché privé}

En entretien, le responsable des marchés d'une commune de la Réunion propose d'expérimenter le critère du risque mortel comme critère de choix dans un marché public. Les discussions avec le tribunal administratif, le conseil d'État et le conseil de l'Europe pourraient avoir lieu sur la base d'un exemple concret, soutenu par un maitre d'ouvrage engagé. Cette proposition pourrait se décliner en marché privé où les règles de commande sont moins contraignantes. La réalisation d'un bâtiment respectueux des droits de tous les hommes permettrait de prouver la faisabilité d'une telle construction. Médiatisé, ce prototype pourrait donner envie à d'autres acteurs de s'engager.

Un deuxième type d'expérimentation pourrait concerner la transformation profonde de la filière de production de la céramique sanitaire en favorisant le recyclage d'éléments sanitaires hors d'usage sur le territoire (Schau, Traverso, Finkbeiner, 2012). Appliqué à l'île de la Réunion où il n'y a actuellement pas d'usine de fabrication de céramique, cela signifierait la mise en place d'une petite unité de production capable de traiter les déchets céramiques du bâtiment et de les transformer en éléments neufs. La question éthique liée au risque d'accident mortel posée au départ de l'article est alors remplacée par des questions liées aux circuits courts et à l'autonomie d'un territoire isolé insulaire.

\section{Conclusion}

Le sujet soulève une question éthique forte que ne se posent actuellement pas les maîtres d'ouvrage, les acheteurs et, de façon générale, les citadins qui, le soir et le matin, utilisent leur salle de bain pour se délasser, se préparer, se défaire des traces de sommeil et s'apprêter à affronter la ville. Cependant, les entretiens préalables menés lors de l'enquête montrent que lorsque la question est posée, son écho est réel. Les personnes interrogées sont troublées et semblent être sincèrement intéressées à trouver un ensemble de solutions viables permettant, à terme, d'envisager un secteur du bâtiment plus propre, qui respecterait autant l'environnement planétaire que la vie humaine de tous, ici et là-bas. De plus, la communauté scientifique s'intéresse de plus en plus à ces sujets. Un numéro spécial de la revue Ressources sur l'analyse sociale du cycle de vie est paru en septembre 2019 (Traverso, Neugebauer, 2019).

Les données analysées dans l'article permettent de porter un discours optimiste sur la possibilité d'un secteur de la construction plus éthique. Il existe déjà des pays qui permettent l'extraction et la fabrication de céramique sanitaire dans le respect des droits de l'homme.

À travers cette étude de cas, la ville est pensée comme un ensemble de matières, de matériaux et de matériels qui résultent de choix en partie inconscients des acteurs de la construction. D'une part, les acteurs, principalement locaux, méconnaissent les différentes filières, souvent opaques, du bâtiment. D'autre part, le temps nécessaire à la recherche d'informations précises ne peut être pris en compte dans une opération de construction qui se joue la plupart du temps contre la montre. La recherche publique, en amont, est alors un lieu de réflexion longue qui doit permettre, à terme, de proposer des outils opérationnels, directement utilisables, aux maîtres d'ouvrage les plus engagés qui souhaitent dépasser le cadre normatif et se rêver citoyens du monde, respectueux des droits de tous les hommes.

En termes de définition, la ville se nourrit du territoire mondial pour se construire dans un contexte normatif de plus en plus exigeant mais encore limité par rapport au respect des droits de l'homme, des citadins et des non-citadins. Chaque ville est le lieu de choix qui se répercutent sur l'ensemble de la population mondiale via les matériaux extraits, transformés et mis en œuvre. Villes et hors- 
villes sont liées dans un même processus de construction qui n'a pour actuelle limite que l'atmosphère planétaire. En termes de réseaux, les villes sont liées entre elles à travers la notion de cycle de vie où les lieux d'extraction et de transformation constituent autant de nœuds participant d'une filière de construction. À chaque nœud se retrouvent pourtant des femmes et des hommes, la nécessité de se loger et de travailler, de vivre en communauté, de bâtir, de grandir. Les films, les romans, les histoires pour enfants racontent le monde. Le secteur du bâtiment pourrait s'en inspirer pour que chacun reprenne conscience de son lien aux autres à travers tous les éléments de son quotidien, du plâtre au mur à l'évier de la salle de bain.

\section{Références}

Benoît NorRis, C. (2013). The methodological sheets for sub-categories in social life cycle assessment, UNEP, SETAC.

Benoît Norris, C. et M. Traverzo (2013). "Health and safety", The methodological sheets for sub-categories in social life cycle assessment, UNEP, SETAC, 116-120.

Bouissou, J. (2015). « Ecocide, l'étain meurtrier », Le Monde, 7 février 2015, récupéré le 30 mai 2019 sur https://www.lemonde.fr/planete/ article/2015/02/07/ecocide-1-etain-meurtrier 4571932 3244.ht$\mathrm{ml}$ ? $\mathrm{xtmc}=$ sable $\_$mafia $\& \mathrm{xtcr}=86$

Coeudevez, C.-S. et S. Déoux (2011). Bâtiments, santé, le tour des labels, Andorre : Médieco.

Commission européEnNe (2007). Document de référence sur les meilleures techniques disponible, fabrication de céramique, Paris, France : Ministère de l'écologie, de l'énergie, du développement durable et de la mer.

GrubERT E. (2018). "Rigor in social life cycle assessment: improving the scientific grounding of SLCA", The international journal of Life cycle assessment, 481-491.

Hämäläinena, P., K. L. SaArela et J. Takala (2009). « Global trend according to estimated number of occupational accidents and fatal work-related diseases at region and country level », Journal of Safety Research, n $40,125-139$
KAdIRI G. (2017). " Au Maroc, les marchands de sable dépouillent les plages », Le Monde, 23 novembre 2017, récupéré le 30 mai 2019 sur https://www.lemonde.fr/afrique/article/2017/11/23/au-marocles-marchands-de-sable-depouillent-les-plages 5218910_3212.ht$\mathrm{ml}$ ? xtmc $=$ sable mafia $\&$ xtcr $=91$

O'Brien M., Doig A. et R. Clift (1996). « Social and environmental life cycle assessment », Journal of Life cycle assessment, 231-237.

Organisation mondiale de la santé (2007). Plan d'action mondial pour la santé des travailleurs, Assemblée mondiale de la santé, WHA60.26, 23 mai 2007.

Pouliquen M. (2009). « Les feldspaths, fiche détaillée », récupéré le 30 mai 2019 sur https://www.lasim.org/images/doc_gratuite/les-feldspaths. pdf

Samuelson P. A. (1975). "Optimum social security in a life-cycle growth model”, International economic review, vol. 16, n³, 539-544.

SAsSEN, S. (1999). « La métropole : site stratégique et nouvelle frontière (Partie 1) », Cultures \& Conflits, 33-34 | printemps-été 1999, 123133.

Schau E. M., Traverso M. et Finkbeiner M. (2012), "Life cycle approach to sustainability assessment: a case study of remanufactured alternators", Journal of Remanufacturing, décembre 2012, récupéré le 30 mai 2019 sur https://link.springer.com/article/10.1186/2210-4690-2-5

Stellman, J. M. (2000). Encyclopédie de sécurité et de santé au travail, 3 éd. Française, Genève, Suisse : Bureau international du travail, volume

Traverso, M. et Neugebauer, S. (2019), «Social Life Cycle AssessmentThe Implementation in Different Sectors», Ressources, special issue, aout-septembre 2019, récupéré le 26 octobre 2019 sur https:// www.mdpi.com/journal/resources/special_issues/social_LCA

Usine NOUVELLE (1999). « Mobilier sanitaire : Roca en course pour le leadership mondial », Usine nouvelle, 16 septembre 1999, récupéré le 30 mai 2019 sur https://www.usinenouvelle.com/article/mobiliersanitaire-roca-en-course-pour-le-leadership-mondial.N94025

VALo, M. (2013). " Le sable, enquête sur une disparition », Le Monde, 28 mai 2013, récupéré le 30 mai 2019 sur https://www.lemonde. fr/culture/article/2013/05/28/le-sable-enquete-sur-une-disparition $34169643246 . \mathrm{html}$ 
